\title{
Differential Conductimetry in Flow Injection. Determination of Ammonia in Kjeldahl Digests
}

\author{
Jarbas José Rodrigues Rohwedder and Celio Pasquini* \\ Instituto de Quimica, Universidade Estadual de Campinas, CEP 13081, C.P. 6154, Campinas, São Paulo, \\ Brazil
}

\begin{abstract}
A differential conductivity meter has been constructed for use in flow injection (FI) systems. The instrument employs a Wien bridge to generate a sinusoidal alternating electrical potential $(2 \mathrm{~V}, 1.4 \mathrm{kHz})$ applied to a measurement bridge containing two twin conductance flow cells. The difference in the conductance between the cells is monitored. The instrument can be used to follow the small changes in conductance that occur in samples in which a high background ionic concentration is found. This facility was used for the determination of ammonia in Kjeldahl digests. The de-ionized water acceptor stream, previously used in the $\mathrm{FI}$ system, was replaced by a $5 \times 10^{-4} \mathrm{~mol} \mathrm{dm}^{-3} \mathrm{HCl}$ solution and the decrease in the conductance caused by the reaction of hydrogen ions with ammonia was monitored. Linear calibration graphs were obtained in the range $0.5-25$ mmol dm-3 of ammonium ion and samples can be processed at a rate of $90 \mathrm{~h}^{-1}$. The relative standard deviation for the peak heights of the Fl signals is $1 \%$ or lower. The differential system was briefly studied for the determination of carbon dioxide by using $\mathrm{NaOH}$ solution as the acceptor stream.
\end{abstract}

Keywords: Differential conductivity meter; ammonia determination; flow injection; nitrogen in Kjeldahl digests; carbon dioxide determination

Conductimetry has recently been used together with flow injection (FI) systems for the determination of compounds that can be converted into a molecular form and diffuse through a polytetrafluoroethylene (PTFE) membrane. ${ }^{1-4}$ The diffused species is received in a de-ionized water stream where it ionizes to a certain extent causing an increase in conductance. The method is very suitable for the determination of ammonia and carbon dioxide, but it was desirable that some of the shortcomings observed should be eliminated.

The calibration graphs for the determination of ammonia and carbon dioxide are not linear because these substances are weak electrolytes in aqueous solution. An exponentially decreasing curve is obtained for the change in the conductance caused by the increase in the concentration of the analyte in the sample. ${ }^{1}$ An additional number of standard solutions should then be used in the calibration procedure if a wide range of concentrations are expected in the samples. This problem could be overcome if the change in the conductance was caused by a reaction which could quantitatively convert the diffused species into a product causing a change in the total ionic mobility of the solution. Further, the advantage of low-cost instrumentation, necessary to conductimetric methodology, ${ }^{1-4}$ is partly negated by the requirement for the use of good quality de-ionized water as the acceptor stream.

It is, therefore, desirable to work with acceptor streams that could contain a high concentration of ions in order to apply selective and quantitative reactions to the diffused species or simply to allow for the use of water of lower quality.

Commercial conductivity meters have a restricted use because they are usually designed to work with only one flow cell. The use of solutions with high ionic concentrations requires the use of a high range conductance scale in the instrument. Small changes cannot be observed owing to the high signal attenuation imposed. Further, small changes in the conductance cannot be amplified as these will take place over a high background signal.

In order to find a solution to these problems, a differential conductivity meter was constructed. The instrument was used to amplify the difference in the conductance between two twin flow cells in an FI manifold which was employed for the determination of ammonia in Kjeldahl digests. This paper

* To whom correspondence should be addressed. describes the instrument, the construction of the conductance flow cells and the methodology for the determination of ammonia.

\section{Experimental}

\section{Differential Conductivity Meter}

Fig. 1 depicts an electronic diagram of the differential conductivity meter. The circuit was supplied by a standard d.c., $\pm 12 \mathrm{~V}, 250 \mathrm{~mA}$ power supply and can be divided into three main parts.

The first part is the source for the a.c. sinusoidal signal applied to the conductance measurement bridge. This unit is based on a Wien bridge. 5 The values selected for the capacitors and resistors allow the bridge to oscillate at a frequency of $1.4 \mathrm{kHz}$. The signal amplitude can be adjusted by the variable resistor $P_{1}$ and a value of $2 \mathrm{~V}$ (peak-to-peak) was chosen for the experiments described here. The frequency of the sinusoidal signal can be easily modified by changing the passive elements of the Wien bridge. The operational 741 amplifier $\left(\mathrm{OP}_{1}\right)$ employed can couple with frequencies in the range $50 \mathrm{~Hz}-50 \mathrm{kHz}$ for a $2 \mathrm{~V}$ peak-to-peak signal amplitude. The second operational 741 amplifier $\left(\mathrm{OP}_{2}\right)$ is simply a buffer stage to supply a current to the bridge. The maximum output current from this unit is about $20 \mathrm{~mA}$ as specified for the 741 amplifier.

The second part is the measurement bridge associated with an instrumentation amplifier used to acquire the differential signal between the two bridge arms. One of the arms, which contains the cell $\mathrm{C}_{1}$, is the reference. The solution flowing through this cell must have a constant ionic composition. The other arm is the indicator arm and it contains the cell $C_{2}$ used to sense any change in the ionic composition of the fluid during the determination procedure. The difference in the electrical voltage between the arms is monitored by the instrumentation amplifier ${ }^{6}$ constructed using 3140 field-effect transistor (FET) operationals $\left(\mathrm{OP}_{3}, \mathrm{OP}_{4}\right.$ and $\left.\mathrm{OP}_{5}\right)$. The voltage difference gain can be adjusted with the potentiometer $\mathrm{P}_{4}$. This stage also contains a circuit for the adjustment of the bridge sensitivity and for finding the balance point. The circuit is based on another 741 amplifier $\left(\mathrm{OP}_{6}\right)$, this time used as a comparator, ${ }^{6}$ and on a simple rectifier circuit. Firstly, the user must adjust the maximum sensitivity by switching the circuit to point $\mathrm{A}$. A comparison between the voltage at the reference arm of the 


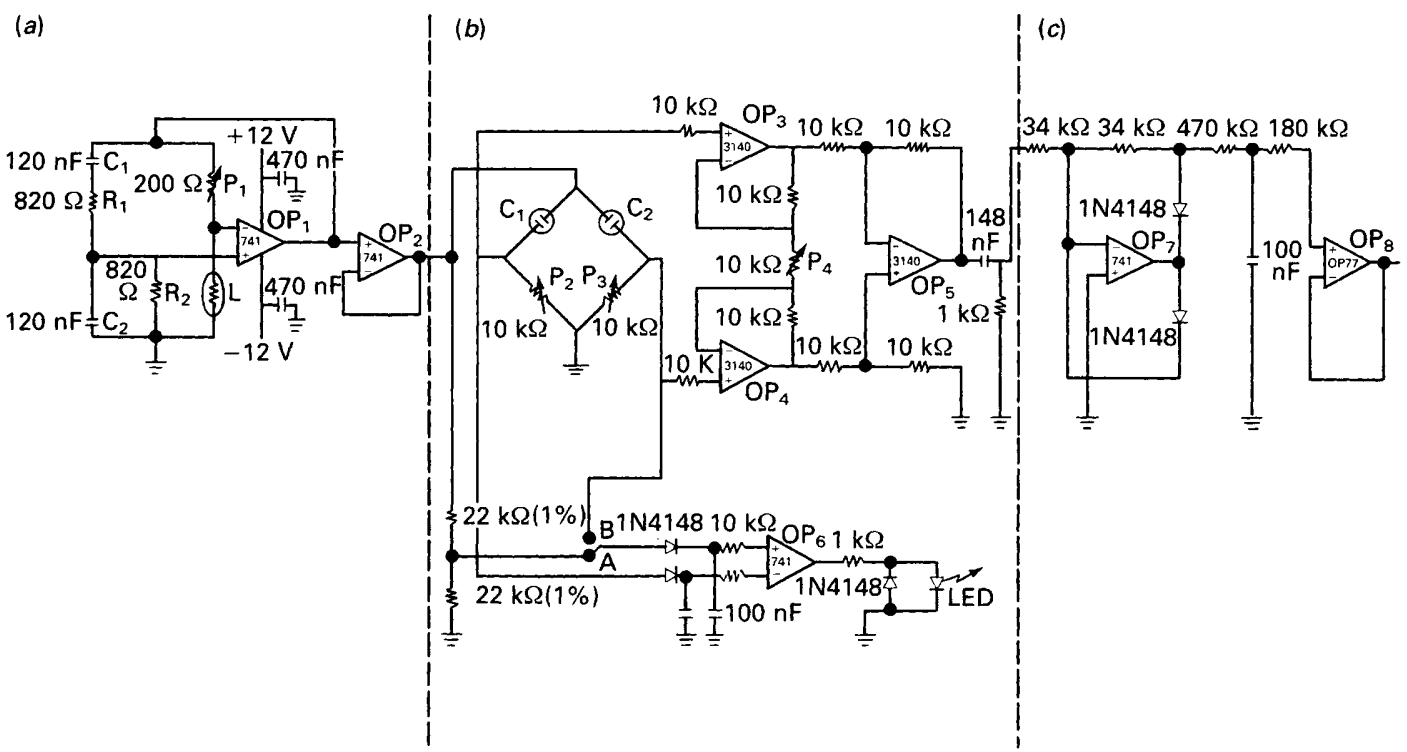

Fig. 1 Circuit diagram of the differential conductivity meter. (a) Wien bridge; $(b)$ measurement bridge with instrumentation amplifier and circuit for sensitivity and balance adjustment; and $(c)$ precision rectifier and output buffer. For details see text

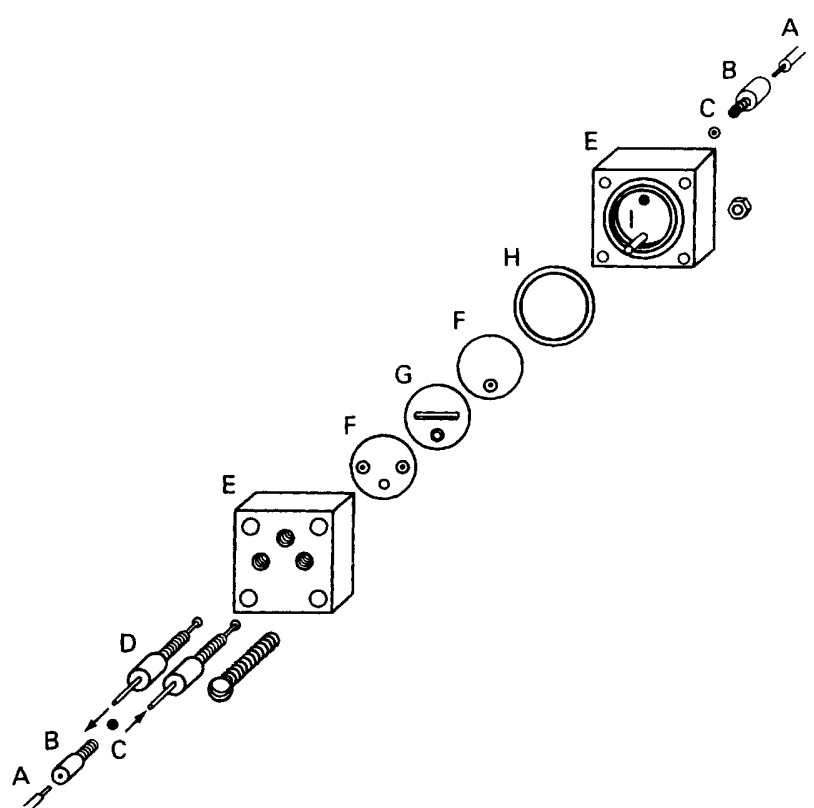

Fig. 2 Conductimetric flow cell: A, electrical contact wire; $\mathbf{B}$, screw for coupling contact wires; C, O-ring; D, screws for coupling the FI manifold tubing; $E$, acrylic block; $F$, stainless-steel disc electrode; $G$, rubber spacer; $\mathrm{H}$, O-ring for preventing contact of the electrodes with the thermal equilibration bath; and I, mounting guide pin

bridge with half of the amplitude of the sinusoidal signal applied to the bridge can be accomplished. The potentiometer $P_{2}$ is adjusted until the light emitting diode (LED) is on, if it was previously off, or, otherwise, until the LED is off. The balance point can then be found by switching to point $B$, comparing the voltage in the reference arm with that in the indicator arm. Potentiometer $P_{3}$ is now utilized; the same LED and its state (on/off) are used to indicate the equilibrium condition. The capacitor values used for this stage are suitable for the working frequency of $1.4 \mathrm{kHz}$.

Finally, the third part consists of a circuit based on another 741 amplifier $\left(\mathrm{OP}_{7}\right)$, capable of rectifying the a.c. signal obtained from the previous stage, avoiding the loss of small signals caused by the diode voltage drop. ${ }^{6}$ The rectified signal is then sent to a final buffer amplifier $\left(\mathrm{OP}_{8}\right)$ and from there to an external signal monitor such as a chart recorder.

\section{Flow Cells}

Fig. 2 shows how the twin flow cells were constructed. The stainless-steel electrodes and the contact wires are housed inside two acrylic blocks, avoiding contact with the water of the isolated water-bath used in the FI manifold. Essentially, the cell constant is determined by a spacer $(G)$ placed between the two metallic discs. The spacer used here was a $3 \mathrm{~cm}$ diameter styrene-butadiene rubber disc, $0.5 \mathrm{~mm}$ thick, with a central hole $20 \mathrm{~mm}$ long and $2 \mathrm{~mm}$ wide. It is very easy to reproduce the spacer dimensions. Therefore, it is also easy to construct cells with matching cell constants.

\section{Other Apparatus and FI Manifold}

The FI manifold used for the determination of nitrogen in Kjeldahl digests is illustrated in Fig. 3. It was constructed using polyethylene tubing of $0.8 \mathrm{~mm}$ i.d. An Ismatec MP13 GJ4 peristaltic pump and Tygon pumping tubes were used. A 51 isolated water-bath was used to allow a long period of operation, independent of the external temperature. ${ }^{1}$ The same diffusion unit previously described, using the same commercial PTFE tape, was also used ${ }^{1}$ for the determination of ammonia. The FI manifold employed for carbon dioxide was the same as that for ammonia, except that it had an enlarged diffusion cell similar to that described previously. ${ }^{3}$

The conductimetric FI signals were registered on an ECB potentiometric reccrder.

\section{Reagents, Samples and Standard Solutions}

All reagents and standard solutions were prepared using freshly de-ionized water. Ammonium standard solutions were prepared daily by suitable dilution of a $0.05 \mathrm{~mol} \mathrm{dm}^{-3}$ ammonium sulphate stock standard solution. Ammonium standard solutions used for the analysis of Kjeldahl digests were prepared so that they had the same sulphuric acid composition as the samples subjected to the digestion procedure. Therefore, these solutions were $1.44 \mathrm{~mol} \mathrm{dm}^{-3}$ in sulphuric acid. Carbonate standard solutions were prepared daily by suitable dilution with de-ionized water from a $0.1 \mathrm{~mol} \mathrm{dm}^{-3}$ sodium carbonate stock standard solution.

Samples of vegetable tissues were provided by the Instituto Agronomico de Campinas and were subjected to the following digestion procedure. To $200 \mathrm{mg}$ of the dry material in a digestion flask were added $8 \mathrm{ml}$ of concentrated sulphuric acid and $2 \mathrm{~g}$ of a mixture prepared to contain $100 \mathrm{~g}$ of potassium 


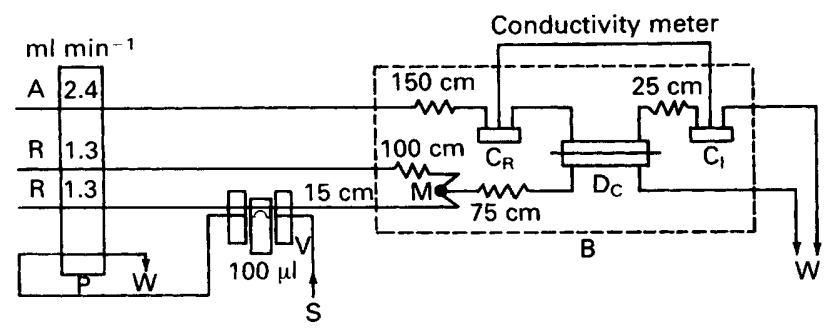

Fig. 3 Flow injection manifold for the determination of nitrogen in Kjeldahl digests: A, acceptor stream $\left(5 \times 10^{-4} \mathrm{~mol} \mathrm{dm}^{-3} \mathrm{HCl}\right) ; \mathbf{R}$, reagent streams $\left(3.0 \mathrm{~mol} \mathrm{\textrm {dm } ^ { - 3 }} \mathrm{NaOH}\right.$ containing $1 \%$ ethylenediaminetetraacetic acid); $\mathbf{P}$, peristaltic pump; $\mathbf{W}$, waste line; $\mathrm{V}$, sample introduction valve; $S$, sample inlet; $M, 30^{\circ}$ mixing point; $C_{R}$, reference flow cell; $C_{I}$, indicator flow cell; $D_{C}$, gas diffusion cell; and $\mathrm{B}$, isolated water-bath

sulphate, $10 \mathrm{~g}$ of copper sulphate and $1 \mathrm{~g}$ of selenium. The flask was placed in a digestion block for $4 \mathrm{~h}$. After digestion, the flask was cooled to ambient temperature and the volume made up to $100 \mathrm{ml}$.

\section{Results and Discussion}

The differential flow conductivity meter was first evaluated in relation to the equality of the reference and indicator arms. By following the signals from the two arms, with the help of an oscilloscope, it was observed that both the signals were in the same phase. This shows that there is a very good similarity between the capacitance component of the two cells and that of the two bridge arms. Small phase displacement was observed only when relatively very concentrated ionic solutions were pumped through the cells. At the selected $\mathrm{HCl}$ or $\mathrm{NaOH}$ solution concentration used, the signals were always in the same phase. Therefore, no capacitance adjustment was required, although the instrument can, if necessary, be used to apply a small variable capacitance in parallel with the indicator cell to correct for a small phase shift. The difference in the resistance of the two cells, when a $5.0 \times 10^{-4} \mathrm{~mol} \mathrm{dm}^{-3} \mathrm{HCl}$ solution is pumped through, is $0.8 \%$, the values for the resistance being 394 and $397 \Omega$.

The performance of the circuit used to find the maximum sensitivity and the balance point of the measurement bridge was also evaluated. It was observed that the circuit could be used to find the maximum sensitivity condition accurately and the balance point could be reached with a maximum difference of $3.0 \mathrm{mV}$ in relation to the true balance point as shown by the oscilloscope.

The drift and noise of the baseline when a $5.0 \times$ $10^{-4} \mathrm{~mol} \mathrm{dm}{ }^{-3} \mathrm{HCl}$ solution was pumped through the system at a rate of $2.4 \mathrm{ml} \mathrm{min}^{-1}$ with the cells immersed in the isolated water-bath of the manifold shown in Fig. 3 were estimated to be $0.3 \mathrm{mV} \mathrm{h}^{-1}$ and $0.01 \mathrm{mV}$, respectively.

As the bridge employed in the measurement circuit has a restricted linear range of response in relation to the change in the resistance of the indicator flow cell, it was necessary to find the range of the output voltage given by the circuit which was directly proportional to the cell resistance. The minimum gain was utilized in the conductivity meter. The flow cells were replaced by potentiometers and the resistance was adjusted to the same value in order to resemble the resistance of the cells when filled with de-ionized water or $5 \times 10^{-4} \mathrm{~mol} \mathrm{dm}^{-3} \mathrm{HCl}$. The potentiometers were then set to various values simulating the changes observed in real measurements (increasing the resistance when $\mathrm{HCl}$ was used as the acceptor stream or decreasing the resistance when de-ionized water was employed instead). The results show that, under the above conditions, the instrument output voltage is linearly related to the change in the resistance in the range $0-80 \mathrm{mV}$. This change in the output voltage corresponds to about a $40 \%$ increase or decrease of the cell resistance in relation to the value observed
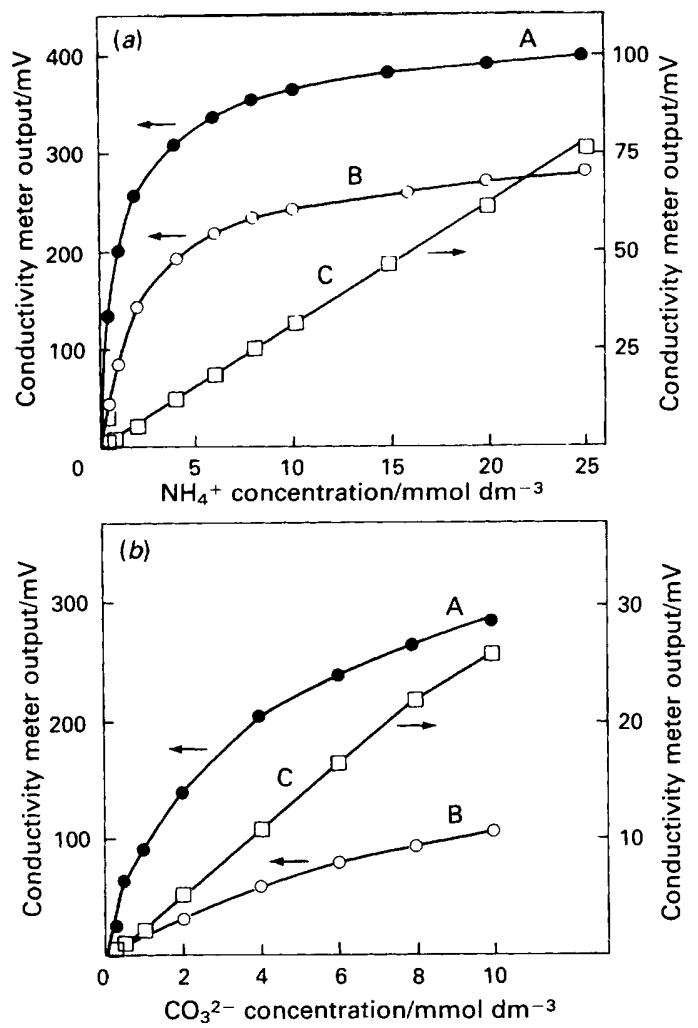

Fig. 4 Calibration graphs for the determination of $(a)$ ammonium and $(b)$ carbonate using several acceptor streams in the differential system. A, De-ionized water; B, distilled water; and C, $5 \times 10^{-4}$ mol dm ${ }^{-3} \mathrm{HCl}$ solution for ammonium and $1 \times 10^{-3} \mathrm{~mol} \mathrm{dm}^{-3} \mathrm{NaOH}$ solution for carbonate

when the bridge is initially balanced. Beyond this limit, the output voltage shows a positive or negative deviation from linearity with a further decrease or increase of the resistance, respectively.

Fig. 4 presents calibration graphs obtained for ammonium and carbonate standard solutions employing various acceptor streams in the FI manifold shown in Fig. 3. The reagent stream for carbonate was a $0.5 \mathrm{~mol} \mathrm{dm}-3$ sulphuric acid solution. It can be seen that the use of a strong electrolyte $(\mathrm{HCl}$ or $\mathrm{NaOH})$ which can promote a quantitative reaction with the diffused species can effectively linearize the calibration graph. The sensitivity of the measurement is, however, decreased as the change in relative conductance taking place in the acceptor stream is lower for a stream that contains an initially high ionic concentration than for that containing de-ionized or distilled water. Nevertheless, the conductance measurements, in the concentration range of interest for the Kjeldahl digests, can be obtained even for a minimum gain of the conductivity meter with very good reproducibility.

The sensitivity for the determination of carbonate is lower than that for ammonia. In order to increase the analytical signal for carbonate, a large diffusion cell was employed. The lower sensitivity observed could be attributed to a smaller decrease in the conductance caused by the decrease of the $\mathrm{OH}^{-}$concentration in the acceptor solution, or to a decrease in the permeation of carbon dioxide through the membrane, or to the low rate of hydration of carbon dioxide. However, further studies are necessary in order to establish the relative contribution of each factor.

The advantages of linearization are mainly related to the small number of standard solutions required in the calibration procedure and to the data treatment which can be carried out very easily. The linear range of the calibration graphs can be increased to higher concentrations by increasing the concen- 


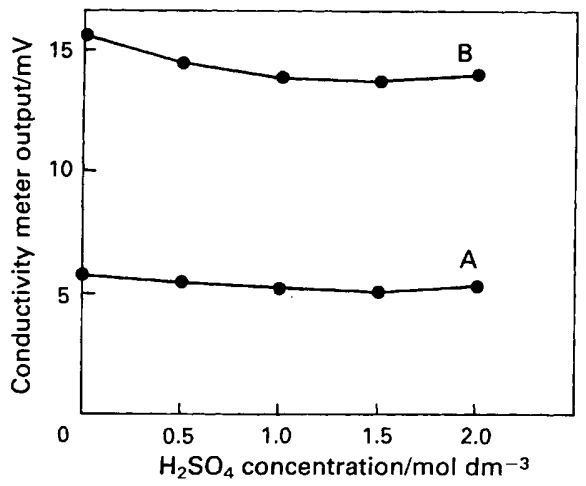

Fig. 5 Effect of the sulphuric acid concentration on the peak height of the conductimetric signal of ammonium standard solutions. Ammonium concentration: A, 2.0; and B, $5.0 \mathrm{mmol} \mathrm{dm}^{-3}$

tration of $\mathrm{HCl}$ or $\mathrm{NaOH}$ in the acceptor stream. The upper limit of the linear range is determined by the acceptor concentration which, for ammonium in the FI manifold used here, was estimated to be approximately two hundredths of the highest ammonium concentration.

The most suitable acceptor stream for the determination of low ammonia and carbonate concentrations is de-ionized water as it can provide very good sensitivity and because the calibration graphs tend to exhibit linear ranges when the concentration of the diffused species is reduced. ${ }^{3}$ The differential conductivity meter allows, however, the use of distilled water as the acceptor fluid with a decrease in the sensitivity that is still acceptable. The previous requirements for good quality de-ionized water and for an in-line polishing column can then be relaxed.

The procedure for the determination of ammonia in Kjeldahl digests ${ }^{1}$ was re-evaluated for differential conductance measurements. The conductance change in this instance is due to the decrease in the total ionic mobility of the acceptor stream caused by the net reaction:

$$
\mathrm{H}^{+}(\mathrm{aq})+\mathrm{NH}_{3}(\mathrm{aq}) \rightarrow \mathrm{NH}_{4}+(\mathrm{aq})
$$

The effect of various substances present in Kjeldahl digests was investigated. Copper sulphate $\left(0.8-5 \mathrm{gl}^{-1}\right)$, selenium $\left(0.1-0.4 \mathrm{~g} \mathrm{l}^{-1}\right)$ and potassium sulphate $\left(10-40 \mathrm{gl}^{-1}\right)$ had no significant effect on the peak height obtained for 2 and $5 \mathrm{mmol} \mathrm{dm}^{-3}$ ammonium standard solutions containing $1.4 \mathrm{~mol} \mathrm{dm}^{-3}$ sulphuric acid.

The results of changing the sulphuric acid concentration are shown in Fig. 5. This is the most important parameter that has to be considered in order to ensure good accuracy for the determination. This is because the analytical signal is partly affected by the acid concentration and because the acid is lost in different amounts using the various digestion procedures. The titration of eight solutions obtained from the standard digestion procedure described under Experimental showed that the acid concentration can range from 1.40 to $1.42 \mathrm{~mol} \mathrm{dm}^{-3}$.

An investigation into the effect of the acid content at a concentration ten times lower than in the original sample showed that its effect is negligible in the range 0.1$0.25 \mathrm{~mol} \mathrm{dm}^{-3}$. An alternative procedure could then be used to overcome the effect of changing the acid concentration of the sample in those situations where the final acid concentration is not known or cannot be maintained in a narrow range. In this procedure, the ammonium standard solutions should be prepared to be $0.14 \mathrm{~mol} \mathrm{\textrm {dm } ^ { - 3 }}$ in sulphuric acid and the samples should be diluted ten times before being injected into the FI manifold.

Fig. 6 shows a typical calibration run for the determination of ammonium in Kjeldahl digests. The precision of the signals and the stability of the baseline can be evaluated. The samples can be processed at a rate of $90 \mathrm{~h}^{-1}$ with negligible carryover.

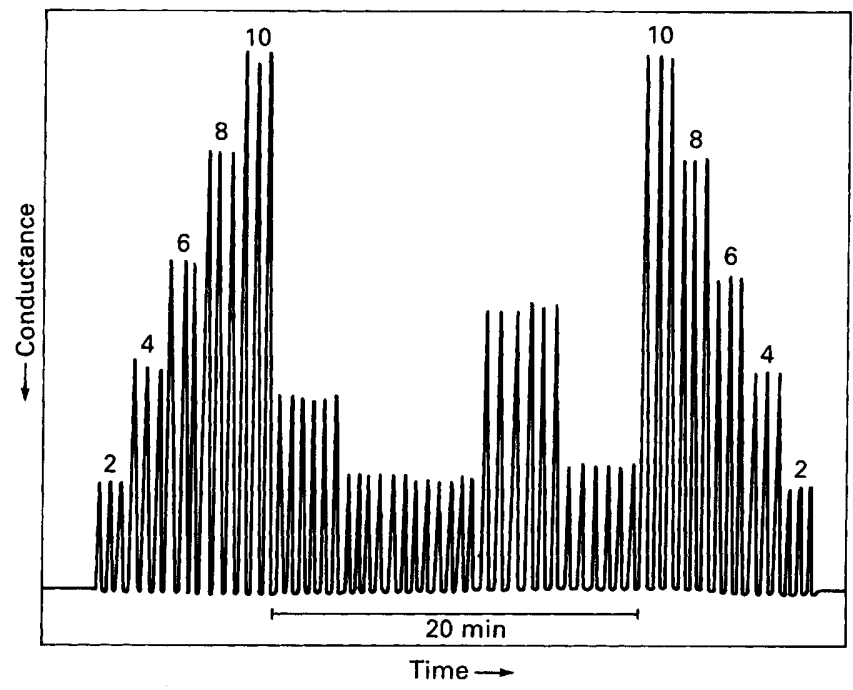

Fig. 6 Typical run for the analysis of Kjeldahl digests. From left to right are shown the signals for five ammonium standard solutions, the signals for ten samples and the signals for the same ammonium standards in reverse order. The numbers over the peaks are the ammonium concentrations in the standard solutions in $\mathrm{mmol} \mathrm{dm}^{-3}$. All measurements were made in triplicate

Table 1 Comparative results for the determination of nitrogen in Kjeldahl digests of plant tissue samples

\begin{tabular}{ccc} 
& \multicolumn{2}{c}{ Nitrogen $/ \mathrm{mmol} \mathrm{kg}^{-1}$} \\
\cline { 2 - 3 } Sample & Interlaboratory* & Proposed method $\dagger$ \\
1 & $314 \pm 22$ & $311 \pm 19$ \\
2 & $1769 \pm 69$ & $1720 \pm 69$ \\
3 & $1094 \pm 55$ & $1080 \pm 21$ \\
4 & $1092 \pm 46$ & $1047 \pm 21$ \\
5 & $2379 \pm 99$ & $2398 \pm 72$ \\
6 & $1224 \pm 41$ & $1206 \pm 19$
\end{tabular}

* Best mean results of 72 interlaboratory determinations together with the standard deviation. ${ }^{7}$

$\uparrow$ Results are expressed together with the calculated standard deviation of five measurements. For details see text.

The results for the determination of the nitrogen content in six samples of vegetable tissues are shown in Table 1 . The nitrogen content in the digested samples was determined five times by employing the proposed conductimetric method using five calibration graphs obtained on different days over a period of 1 week. For comparison purposes the same number of significant figures found in an interlaboratory report ${ }^{7}$ were used when expressing the results of the conductimetric method. The correlation between the results obtained with the conductimetric method and the average of 72 interlaboratory values reported for the samples ${ }^{7}$ can be expressed by: $c_{\mathrm{c}}=$ $(-26.6 \pm 0.9)+(1.006 \pm 0.018) c_{\mathrm{i}}$, where $c_{\mathrm{c}}$ is the nitrogen concentration in $\mathrm{mmol} \mathrm{kg} \mathrm{kg}^{-1}$ found by the proposed conductimetric method and $c_{\mathrm{i}}$ that for the interlaboratory results. The correlation coefficient is 0.9993 and the error of estimate is $28.3 \mathrm{mmol} \mathrm{kg} \mathrm{kg}^{-1}$. The mean results for the conductimetric method show a slight negative systematic error which can be attributed to the digestion procedure. In fact, the results of standard additions, carried out to increase the ammonium concentration of the sample solutions in increments of $1.0 \mathrm{mmol} \mathrm{dm}^{-3}$, showed a mean recovery of $101.0 \%$. This demonstrates that there is no matrix effect on the conductimetric method.

\section{Conclusion}

The differential conductivity meter described in this work can improve the determination of molecular species such as 
ammonia and carbon dioxide, after their diffusion through a membrane. The main advantage is the linearization of the calibration graph.

Selective reactions are therefore possible in both sides of the FI manifold, which might lead to the development of new and more selective $\mathrm{FI} /$ conductimetric methodologies.

Further, the differential conductivity meter could be used to extend the conductimetric determinations directly to samples with a high background ionic concentration. In order to perform this type of determination the signal-to-noise ratio should be improved in order to measure the very small conductance changes that will be observed.

The authors are grateful to $\mathrm{O}$. C. Bataglia for providing the plant tissue samples.

\section{References}

1 Pasquini, C., and de Faria, L. C., Anal. Chim. Acta, 1987, 193, 19.
2 Jardim, W. F., Pasquini, C., Guimarães, J. R., and de Faria, L. C., Water Res., 1990, 24, 351.

3 de Faria, L. C., and Pasquini, C., Anal. Chim. Acta, 1991, 245, 183.

4 de Faria, L. C., Pasquini, C., and Neto, G. O., Analyst, 1991, 116, 357.

5 Horowitz, P., and Hill, W., The Art of Electronics, Cambridge University Press, Cambridge, 8th edn., 1987.

6 Malcolme-Lawes, D. J., Microcomputers and Laboratory Instrumentation, Plenum Press, New York, 2nd edn., 1988.

7 International Plant-Analytical Exchange, Department of Soil Science and Plant Nutrition, Wageningen Agricultural University, The Netherlands, Bimonthly Report 90.3 (May-June), 1990, p. 13 